\title{
Automation of learning-set testing: The video-task paradigm
}

\author{
DAVID A. WASHBURN, WILLIAM D. HOPKINS, and DUANE M. RUMBAUGH \\ Georgia State University, Atlanta, Georgia
}

\begin{abstract}
Researchers interested in studying discrimination learning in primates have typically utilized variations in the Wisconsin General Test Apparatus (WGTA). In the present experiment, a new testing apparatus for the study of primate learning is proposed. In the video-task paradigm, rhesus monkeys (Macaca mulatta) respond to computer-generated stimuli by manipulating a joystick. Using this apparatus, discrimination learning-set data for 2 monkeys were obtained. Performance on Trial 2 exceeded $80 \%$ within 200 discrimination learning problems. These data illustrate the utility of the video-task paradigm in comparative research. Additionally, the efficient learning and rich data that were characteristic of this study suggest several advantages of the present testing paradigm over traditional WGTA testing.
\end{abstract}

The Wisconsin General Test Apparatus (WGTA), or some variation thereof, has been the standard testing apparatus for studying primate learning for over 3 decades. The WGTA is a manual apparatus that presents subjects with discriminative stimuli-objects placed upon stimulus trays. Responses are observed by the experimenter through a one-way screen and are manually recorded. Using the WGTA, Harlow $(1949,1951)$ pioneered the study of primate discrimination learning and developed the concept of learning set (LS), which is the transfer of learning from problem to problem, or "learning to learn." Following this seminal research, LS data have been collected for numerous primates, including human children, gorillas, chimpanzees, gibbons, rhesus monkeys, squirrel monkeys, and various other monkey and prosimian species (for reviews of these studies, see Fobes \& King, 1982; Meador, Rumbaugh, Pate, \& Bard, 1987). In addition, discrimination LS data have been obtained for a variety of nonprimate species (Fobes \& King, 1982). Although the details in these studies might have differed, in each one, some form of the WGTA was employed to assay learning abilities and to compare learning across species (see Levine, 1959, for a discussion of LS theory). So significant was this apparatus to comparative psychology that, in a recent review of primate learning research, Fobes and King (1982) observed that the findings of primate discrimination learning studies are inseparable from the use of the WGTA.

The research reported here was supported by Grant NAG2-438 from the National Aeronautics and Space Administration. Additional support was provided by Grant NICHD 06016 from the National Institutes of Health. Preparation of this manuscript was facilitated by use of the Allan M. Schrier Commemorative Library of the Language Research Center, Georgia State University, Atlanta, Georgia. The authors gratefully acknowledge the theoretical and technical contributions of $E$. Sue Savage-Rumbaugh and W. Kirk Richardson to this research. Reprint requests may be sent to David A. Washburn, Department of Psychology, Georgia State University, Atlanta, Georgia 30303.
With advances in technological complexity and affordability, the WGTA has been modified and automated. Sidowski and Spears (1970), for instance, proposed an automated apparatus to test learning in human and nonhuman primates (see also Polidora \& Main, 1963; Pribram, Gardner, Pressman, \& Bagshaw, 1963). This type of automation of the WGTA has the potential to save the experimenter a great deal of time and effort. However, such automations of the WGTA have been relatively complex and costly and typically are susceptible to performance disruptions as a function of stimulus-response spatial discontiguity (Meyer, Treichler, \& Meyer, 1965). Learning is compromised, or even denied, when stimuli are removed from response sites, even if only by centimeters. Consequently, modifications of the standard, manually operated WGTA have remained very popular in investigations of primate learning and cognition (e.g., Rumbaugh, Savage-Rumbaugh, \& Hegel, 1987).

The applicability of computers and joysticks to comparative research has been explored in our laboratory. Savage-Rumbaugh (1986) reported that 2 chimpanzees (Pan troglodytes) with extensive language training learned by observation alone to perform special video tasks. From the first trials, the chimpanzees were able to manipulate the joystick to bring a cursor into contact with a computergenerated target.

The ease and facility with which the chimpanzees mastered the demands of the video task encouraged us to employ the same technology with rhesus monkeys (Macaca mulatta), despite the species' well-documented sensitivity to performance disruption by stimulus-response spatial discontiguity (Meyer et al., 1965). Although the stimuli in the video-task paradigm are separated from response and reward sites by at least $20 \mathrm{~cm}$, we successfully trained 2 rhesus monkeys to manipulate the joystick in response to various computer-generated stimuli (see Rumbaugh, Richardson, Washburn, Savage-Rumbaugh, \& Hopkins, 1989, for details of these training procedures). 
Subsequently, a wide variety of questions of cognitive and motor competence were formatted into computeradministered video tasks and presented to the monkeys. Among these questions, we examined the implications of this new paradigm on old issues of discrimination learning and learning set.

In the process, an alternative to the traditional WGTA testing paradigm has been serendipitously developedan alternative that combines the advantages of the earlier automations with the simplicity and relative affordability of the standard apparatus. LS data collected for these animals will be presented as a demonstration and confirmation both of the relatively advanced learning skills of rhesus macaques and of the usefulness of the video-task paradigm as applied to the study of primate learning and cognition.

\section{METHOD}

\section{Subjects}

Two 6-year-old rhesus monkeys (Macaca mulatta) served as subjects. Each was skilled in using a joystick to respond in video-formatted tasks. Details of their previous trai.ing are reported elsewhere (Rumbaugh, Richardson, et al., 1989). The animals were neither food- nor water-deprived throughout this experiment; however, access to food during experimental sessions was generally restricted to that available by appropriate responding.

\section{Apparatus}

The animals were tested in their individual home cages, $75 \times 180 \times 90 \mathrm{~cm}$ wire-mesh structures. The animals were neither chaired nor otherwise restrained. Each cage could be rolled up to a computer testing station that consisted of a Sony 1310 color monitor and a standard analog joystick. The monitor was encased in clear Lexan, with the joystick mounted upon the Lexan such that the handle extended approximately $6 \mathrm{~cm}$ toward the animal. Thus, an animal could reach through the mesh of the cage and manipulate the joystick while looking directly at the monitor.

The monitor and joystick were connected to a Zenith Z-159 computer, which was used to administer test conditions and to record the data. Auditory feedback generated by the computer was played through an external speaker/amplifier (Radio Shack \#32-2031) located above the monitor. To do this, the signal was physically redirected from the internal speaker to the external amplifier. The computer also controlled the delivery of reinforcers, $97-\mathrm{mg}$ banana-flavored chow pellets (Noyes), which were dispensed into pellet cups mounted on a cage wall. Control of the pellet dispensers was mediated by a Metrabyte P10-12 I/0 board and a Metrabyte ERA-01 eight-channel relay board.

\section{Procedure}

The animals were tested on successive two-choice discrimination learning problems using this apparatus. Each trial began with a cursor (a white + approximately $1 \times 1 \mathrm{~cm}$ in size) displayed on the center of the screen. Two monochromatic, planimetric stimuli were also presented on the screen. ${ }^{1}$ The location of each stimulus was determined randomly, with the constraint that each was located at least $5 \mathrm{~cm}$ from the cursor and at least $5 \mathrm{~cm}$ from the other. Thus, within the physical limits of the screen, the targets could occupy a large number of possible locations.

Stimuli were arbitrarily chosen and paired for testing. They included random shapes, letters, numbers, and line drawings, and came in a variety of sizes and fonts. Examples of these stimuli are shown in Figure 1.

One of each pair was arbitrarily designated the positive stimulus. The monkey's selection of the positive stimulus was reinforced with a food pellet; its selection of the negative stimulus resulted only in a raucous buzz.

Until the joystick was moved, and whenever it was released, the screen was static. Manipulation of the joystick caused isomorphic movement of the cursor on the screen, accompanied by auditory feedback, a staccato series of low tones. Thus, an animal could select one of the discriminanda by manipulating the joystick so as to move the cursor into contact with either of the stimuli. Selection of either stimulus caused a trial to end.

Each animal was given 200 two-choice discrimination learning problems. The first 60 of these problems were administered as part of Transfer Index measurement (see Rumbaugh \& Pate, 1984), in which an animal was allowed to continue within a discrimination problem until a preselected criterion was achieved $(67 \%$ or $84 \%)$, at which time the reward values for the two stimuli were switched for 11 trials. The animals reached criterion in an average of approximately 19 trials during these problems. The remaining 140 problems were administered as standard 6-problem tests of learning set. After 6 trials, each problem was terminated and two new discriminanda were presented.

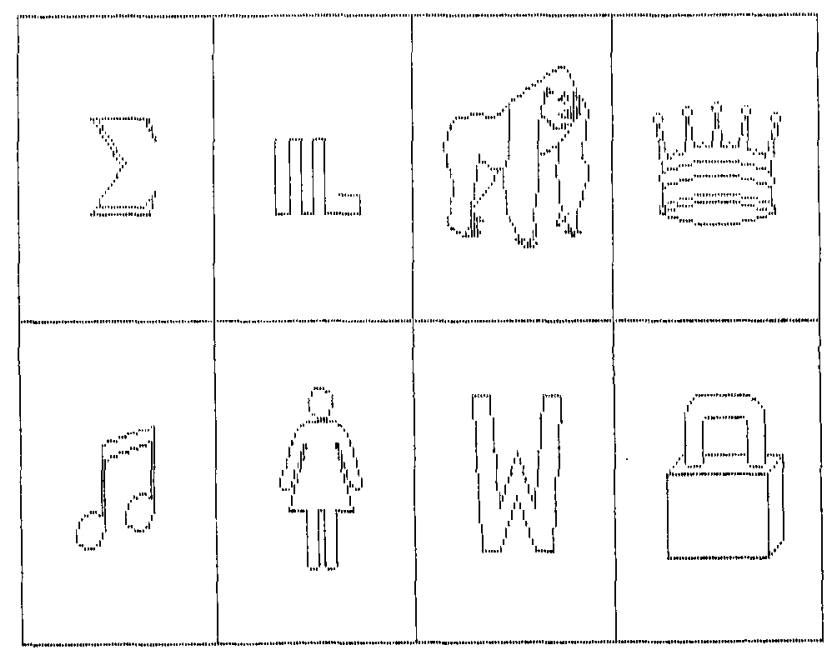

Figure 1. Samples of the stimuli that were arbitrarily paired and presented in this research. 


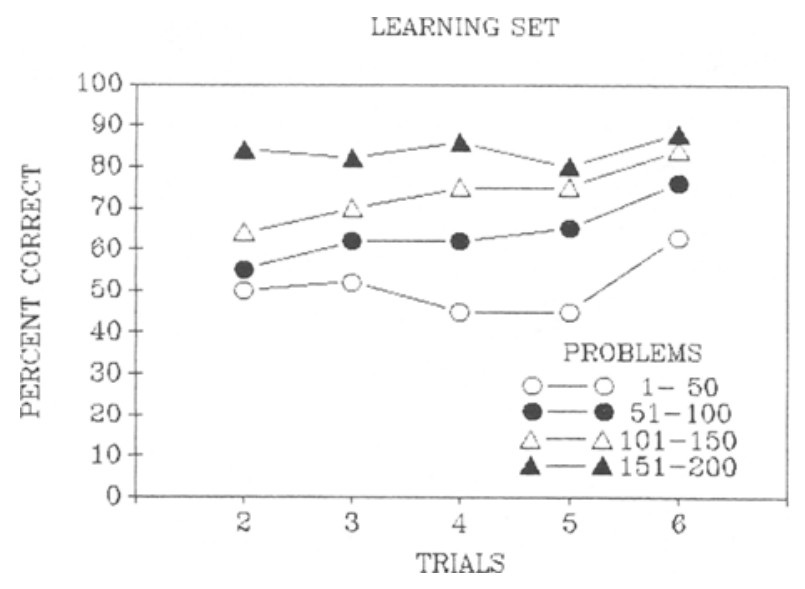

Figure 2. Percentage of correct responses, averaged across animals, for blocks of $\mathbf{5 0}$ two-choice discrimination problems.

\section{RESULTS}

Performance on blocks of 50 two-choice discrimination problems, averaged across animals, is depicted in Figure 2. These data show the development of Trial 2 learning set. Note that performance on Trial 2 is better than $80 \%$ by the last 50 discrimination problems.

\section{DISCUSSION}

The development of Trial 2 learning set in these animals, tested within the video-task paradigm, was comparable to that reported by earlier researchers who employed more traditional WGTA-type apparatus (e.g., Harlow, 1951; Harlow \& Warren, 1952; also see reviews by Fobes $\&$ King, 1982; Meador et al., 1987). The overall level of performance achieved and the number of trials needed to reach this level were well within the range suggested by the literature on the subject. In fact, most investigations of LS in thesus monkeys report that 300 or more problems are necessary before Trial 2 performance exceeds 80\% (Behar, 1962; Miles, 1957), although Harlow's (1951) animals' performance exceeded $90 \%$ after 300 problems.

This rapid development of LS in our animals occurred despite numerous substantive differences between the present work and previous investigations. The fact that the stimuli were planimetric, monochromatic, and inherently spatially discontiguous both with the response site and with the reward site should have impaired, if not prevented, the development of learning set. Notwithstanding, these animals responded in ways congruent with research with literal, three-dimensional discriminanda that were physically touched and manipulated by research animals.

We believe that the video-task paradigm affords a number of advantages to the discrimination-learning testing situation and that these advantages account for the performance of the animals in the present research. The con- sistent and isomorphic movement of images on the screen in concert with manipulation of the joystick, coupled with auditory feedback and clear visual access, certainly influenced the animals' attention to the task and, consequently, their learning efficiency. Movements of the responding hand per se became irrelevant; rather, the cursor and discriminanda on the screen were sampled as the relevant response cues. Additionally, because the absolute locations of the discriminanda were randomly selected, each trial likely attenuated the degree to which the animals could respond on the basis of nonsalient cues (e.g., position).

The video-task paradigm offers numerous advantages in addition to considerations of the efficiency in learning. This paradigm has been employed with success in a variety of configurations (with PC-compatible as well as Commodore computers) and with a variety of subject populations, including mentally retarded and normally developing humans, chimpanzees (Pan troglodytes and Pan paniscus), orangutans (Pongo pygmaeus), and monkeys. The savings in time and effort and the gains in precision and reliability afforded by the application of computer technology to psychological research hardly require enumeration. In short, the testing situation becomes increasingly data-rich and efficient with the application of this technology. Finally, the suggestion that the animals might find the video tasks inherently rewarding and thus might be enriched by the paradigm is, while yet untested, quite possible (see Rumbaugh, Washburn, \& SavageRumbaugh, 1989). Consequently, this electronic transformation and automation of an old, reliable apparatus promises to yield even greater insight into the processes of primate learning and cognition.

\section{REFERENCES}

BEHAR, I. (1962). Evaluation of the significance of positive and negative cue in discrimination learning. Joumal of Comparative \& Physiological Psychology, 55, 502-504.

Fobes, J. L., \& KING, J. E. (1982). Primate behavior. New York: Academic Press.

Harlow, H. F. (1949). The formation of learning sets. Psychological Review, 56, 51-65.

Harlow, H. F. (1951). Primate learning. In C. Stone (Ed.), Comparative psychology (3rd ed., pp. 183-238). New York: Prentice-Hall.

HARLOW, H. F., \& WARREN, J. M. (1952). Formation and transfer of discrimination learning sets. Journal of Comparative \& Physiological Psychology, 45, 482-489.

LEVINE, M. (1959). A model of hypothesis behavior in discrimination leaming set. Psychological Review, 66, 353-366.

Meador, D. M., Rumbaugh, D. M., Pate, J. L., \& Bard, K. A. (1987). Learning, problem solving, cognition, and intelligence. In G. Mitchell \& J. Erwin (Eds.), Comparative primate biology: Behavior, cognition, and motivation (Vol. 2, Part B, pp. 17-83). New York: Liss.

Meyer, D. R., Treichler, F. R., \& Meyer, P. M. (1965). Discretetrial training techniques and stimulus variables. In A. Schrier, H. Harlow, \& F. Stollnitz (Eds.), Behavior of nonhuman primates (Vol. 1, pp. 1-49). New York: Academic Press.

Miles, R. C. (1957). Learning-set formation in the squirrel monkey. Journal of Comparative \& Physiological Psychology, 50, 356-357.

Polidora, V. J., \& MaIN, W. T. (1963). Punched card programming and recording techniques employed in the automation of the WGTA. Journal of the Experimental analysis of Behavior, 6, 599-603. 
Pribram, K. H., Gardner, K. W., Pressman, G. L., \& Bagshaw, M. (1963). Automated analysis of multiple choice behavior. Journal of the Experimental Analysis of Behavior, 6, 123-124.

Rumbaugh, D. M., \& Pate, J. L. (1984). The evolution of cognition in primates: A comparative perspective. In H. L. Roitblat, T. G. Bever, \& H. S. Terrace (Eds.), Animal cognition (pp. 569-587). Hillsdale, NJ: Erlbaum.

Rumbaugh, D. M., Richardson, W. K., Washburn, D. A., SavageRumbaugh, E. S., \& Hopkins, W. D. (1989). Rhesus monkeys (Macaca mulatta), video tasks, and implications for stimulus-response spatial contiguity. Journal of Comparative Psychology, 103, 32-38.

Rumbaugh, D. M., Savage-Rumbaugh, E. S., \& Hegel, M. (1987). Summation in the chimpanzee (Pan troglodytes). Joumal of Experimental Psychology: Animal Behavior Processes, 13, 107-115.

Rumbaugh, D. M., Washburn, D. A, Savage-Rumbaugh, E. S.
(1989). The care of captive chimpanzees: Methods of enrichment. In E. Segal \& P. Scollay (Eds.), Psychological wellbeing of captive primates (chap. 23, pp. 357-375). Park Ridge, NJ: Noyes Publications.

Savage-Rumbaugh, E. S. (1986). Ape language: From conditioned response to symbol. New York: Columbia University Press.

SIDOWSKı, J. B., \& SPEARS, C. A (1970). A versatile apparatus for measuring the frequencies and durations of animal and human responses. Behavior Research Methods \& Instrumentation, 2, 235-238.

\section{NOTE}

1. The stimuli were designed with PC Picture Graphics, a public domain graphics package that allows stimuli to be drawn, or predrawn clip-art to be imported, and saved for later use. Any graphics package that includes the option to "BSAVE" a graphic should work equally well. Contact D. M. Rumbaugh for access to our software programs. 